\title{
Caustic region fields of an elliptical reflector covered by an anisotropic magnetized plasma layer
}

\author{
A. Ghaffar \\ aghaffarr@ksu.edu.sa \\ M. A. S. Alkanhal \\ majeed@ksu.edu.sa
}

\author{
Department of Electrical Engineering, King Saud University, Saudi Arabia \\ Department of Physics, University of Agriculture, Faisalabad, Pakistan \\ Department of Electrical Engineering, King Saud University, Saudi Arabia
}

Theoretical formulations of the electromagnetic (EM) field distribution in the focal or caustic region of an elliptical reflector with its curved surface coated by collisional magnetized anisotropic plasma have been derived. Both parallel and perpendicular polarizations of the normal incident wave are considered. Expressions for the EM field intensity along the focal region have been obtained accurately using Maslov's method. The effect of the plasma-layer thickness on the reflected and transmitted field intensity distributions was investigated. Other physical parameters such as the plasma electron density, the cyclotron frequencies, and the collisional frequency have been noticed to have an evident influence on the levels of the transmitted field-intensities along the focal region. The problem discussed in this paper has, also, been solved using Kirchhoff's approximation and the results of the two methods are found to be in a good agreement.

[DOI: http://dx.doi.org/10.2971/jeos.2015.15034]

Keywords: Wave propagation, plasma layer, plasma frequency, Maslov's method, focal region

\section{INTRODUCTION}

The study of EM wave propagation in isotropic and anisotropic plasma medium has been attracting many researchers for many years. Numerous researches that explored plasma as waveguides, absorbers, and reflectors have been published recently [1]-[4]. Henceforth, deeper understanding of the effects of plasma on the behavior of EM field components is imperative in the current arena of advanced technologies in microwave, millimeter-wave, and optical device applications. Moreover, plenty of research has been produced in the last fifty years related to reflector antennas and their applications by different authors [5, 6]. This noticeable research work discussed many aspects of reflector antennas such as materials, beam patterns, polarizations, efficiencies, and surface errors in order to enhance the performance of such antennas for various frequency bands and application $[7,8]$.

Analysis of focal region field in reflectors is important for many applications like optical spectroscopy, medical treatment and hyperthermia [9]-[11]. High velocity space vehicles with metallic antennas, as reflector antennas for example, encounter plasma effect when re-entering into the Earth's atmosphere. Thus, usually antennas of space vehicles are in surface contact with a plasma layer that affects the radiation characteristics of such antennas [12, 13]. Similarly, the existence of a plasma layer on metallic targets changes the reflected and scattered wave energies. Analysis is very significant to find out the parameters of the plasma, which affect the reflection, absorption, and transmission of the EM energy especially in the study of the interaction of intense EM waves with curved metallic surfaces [14]-[16]. These research activ- ities mostly focused on the propagation of EM waves in unmagnetized plasma. If a magnetic field is applied to the background plasma equilibrium, the plasma will be noticeably anisotropic and the characteristics of the EM waves propagation in the plasma will be affected more dynamically. Recently, several researchers have reported studies relevant to the interaction and propagation of EM waves in magnetized plasma [17]-[20].

Many types of reflectors have been investigated for focusing EM waves. Among different shaped reflectors, an elliptical reflector has not received sufficient investigations like the parabolic reflector. This reflector focuses rays emitted by one focus at the other and vice versa [21]-[23]. An elliptical reflector shape could be generated in a variety of ways: one may refer to the geometrical structures made by the joining of the cylindrical and conic shape with the cone generated curved surface or resulting from the bending of any actual parabolic or circular reflector [24].

This paper considers a long metallic elliptical reflector covered with a magnetized plasma layer with a normal incidence plane-wave for both parallel and perpendicular polarizations. The exact analytical field expressions of the field intensity distribution of reflected wave from curved plasma layer and the transmitted wave out of layer after reflection from metallic elliptical reflector are derived along its caustic or focal point by Maslov's method. Explanations and detailed procedures concerning the application of Maslov's method are found extensively in literature [25]-[27] and many researchers have used this method to study the focusing of the different reflecting 
and transmitting antennas [28]-[33]. In spite of analyzing unmagnetized plasma, the present study facilitate observing the variations of the reflection and absorption of the anisotropic magnetized plasma by the continuously varying parameters such as the cyclotron and collisional frequencies. As a result, the effects of the cyclotron frequency on the electric field intensity in plasma layer can be more visibly described. The effects of plasma layer thickness, electron density and cyclotron frequencies of plasma on the field intensity distribution of reflected wave from curved plasma layer and the transmitted wave out of layer after reflection from metallic elliptical reflector along its caustic or focal point have been analyzed and discussed numerically. The numerical computational comparison of derived expressions by Maslov's method has been confirmed alternatively by using Kirchhoff's approximation. The time-harmonic $(i \omega t)$ dependence is adopted and suppressed in what follows.

\section{FORMULATIONS}

Consider an elliptical reflector made of a perfect metal in the presence of an anisotropic plasma layer exposed to an external uniform magnetic field $B=B_{0} \hat{e}_{z}$ as shown in Figure 1 . It is assumed that an EM plane wave is incident normally on the surface of the reflector parallel to its symmetry axis. The equation of the metallic elliptical reflector is given by

$$
\zeta=a \sqrt{1-\frac{\zeta^{2}}{b^{2}}}
$$

where $a$ is the length of major axis and $b$ is the length of minor axis of perfect conductor elliptical reflector. Then, the surface equation of the anisotropic magnetized plasma layer placed on perfect metal elliptical reflector is given as under

$$
\zeta=(a-d) \sqrt{1-\frac{\xi^{2}}{(b-d)^{2}}}
$$

where $d$ is the thickness of the elliptical anisotropic plasma layer. It is assumed that the coordinates of a point on the elliptical surface of the anisotropic plasma layer $P\left(\xi_{0}, \zeta_{0}\right)$ corresponds to a point on the metallic elliptical reflector $Q(\xi, \zeta)$. The magnetized plasma layer has the relative permittivity in tensor form as

$$
[\epsilon]=\left[\begin{array}{ccc}
\epsilon_{1} & j \epsilon_{2} & 0 \\
-j \epsilon_{2} & \epsilon_{1} & 0 \\
0 & 0 & \epsilon_{3}
\end{array}\right]
$$

The non-zero elements of this tensor are defined under the relations

$$
\begin{aligned}
& \epsilon_{1}=1-\frac{\omega_{p}^{2}(\omega-j v)}{\omega\left[(\omega-j v)^{2}-\omega_{c}^{2}\right]} \\
& \epsilon_{2}=\frac{\omega_{c} \omega_{p}}{\omega\left[(\omega-j v)^{2}-\omega_{c}^{2}\right]} \\
& \epsilon_{3}=1-\frac{\omega_{p}^{2}}{\omega(\omega-j v)}
\end{aligned}
$$

where $\omega$ and $\omega_{c}$ are the incident wave frequency and the cyclotron frequency, $v$ is the effective collision frequency and $\omega_{p}=n_{e} e^{2} / m \epsilon_{0}$ is the plasma frequency which satisfy the

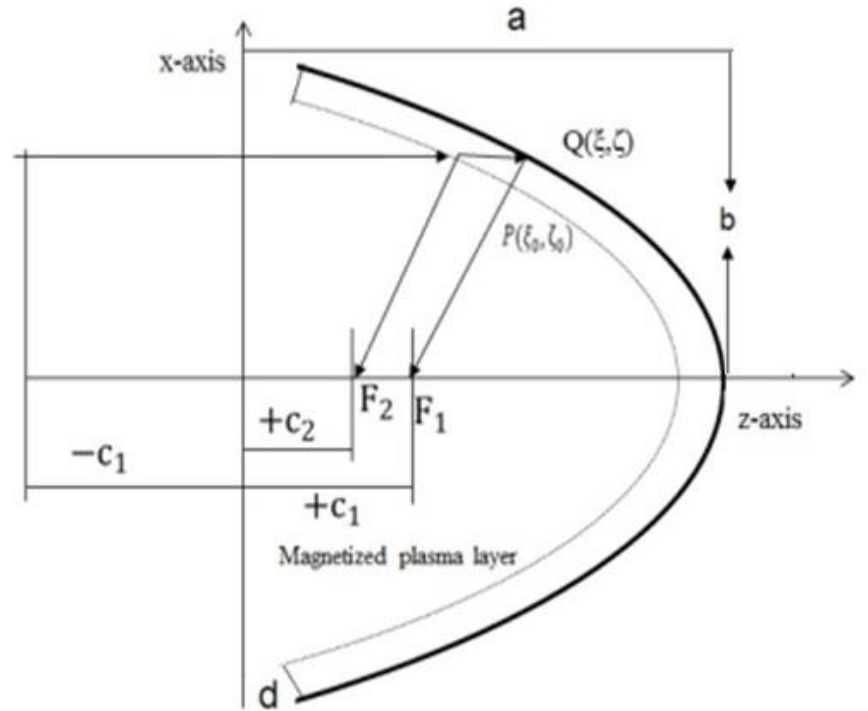

FIG. 1 Elliptical reflector made of perfect metal with a layer of anisotropic plasma on its surface.

condition of under-dense plasma $\omega_{p}<\omega$. Their explicit expressions are found extensively in literature [1]-[3]. The wave numbers in anisotropic plasma for parallel (TE) polarization and perpendicular $(\mathrm{TM})$ polarization are $k_{1}=\sqrt{\epsilon_{3}} k_{0}$, and $k_{2}=\sqrt{\left(\epsilon_{1}^{2}-\epsilon_{2}^{2}\right) / \epsilon_{1}} k_{0}$ respectively.

\subsection{Parallel polarization (TE)}

Now, we consider a parallel polarized monochromatic EM wave incident on an the elliptical anisotropic plasma layer

$$
E_{0 i}=E_{i} \hat{e}_{x} \exp \left[-j k_{i} z\right]
$$

In the anisotropic plasma layer, there are two extra ordinary waves propagating toward the interface $z=\zeta$ and the interface $z=\zeta_{0}$. By using the Snell's laws the reflected field wave vector and the transmitted field wave vector can be obtained in the following

$$
\begin{gathered}
p^{r}=p^{i}-2\left(p^{i} \cdot N\right) N \\
q^{r}=p^{i}+\sqrt{n^{2}-1+\left(p^{i} \cdot N\right)^{2}} N-\left(p^{i} \cdot N\right) N
\end{gathered}
$$

where $p^{i}$ is the wave vector of the incident wave, $n$ is refractive index of the anisotropic plasma medium and $N$ is the unit normal to thesurface of an elliptical reflector coated with plasma layer which can be written as

$$
N=\hat{e}_{z} \cos \alpha+\hat{e}_{x} \sin \alpha
$$

The reflected wave vector $p_{1}$, the refracted wave vector $q_{1}$ and $q_{2}$ from the anisotropic plasma elliptical layer and the reflected wave vector $p_{2}$ from the PEC elliptical reflector are given $[27,28]$ as

$$
p_{1}=-\hat{e}_{x} \sin 2 \alpha-\hat{e}_{z} \cos 2 \alpha
$$

$$
\begin{aligned}
q_{1}=- & \hat{e}_{x} \sin \alpha\left(-\cos \alpha+\sqrt{n_{1}^{2}-\sin ^{2} \alpha}\right) \\
& -\left(\cos \alpha\left(\cos \alpha+\sqrt{n_{1}^{2}-\sin ^{2} \alpha}\right)+1\right) \hat{e}_{z}
\end{aligned}
$$




$$
\begin{gathered}
p_{2}=-\hat{e}_{x} \sin \alpha\left(K_{1}+\cos \alpha\right)-\hat{e}_{z}\left(K_{1} \cos \alpha-\sin ^{2} \alpha\right) \\
q_{2}=\hat{e}_{z}\left(n_{1} \sin ^{2} \alpha-K_{2} \cos \alpha\right)-\hat{e}_{x} \sin \alpha\left(K_{2}+n_{1} \cos \alpha\right)
\end{gathered}
$$

where $K_{2}=\sqrt{\left(2+n_{1}\left(-1+2\left(-1+n_{1}\right) n_{1}+\cos 2 \alpha\right) / 2\right.}$, $K_{1}=\sqrt{\left(-1+2 n_{1}^{2}+\cos 2 \alpha\right) / 2}$ and $n_{1}=\sqrt{\left(\epsilon_{1}^{2}-\epsilon_{2}^{2}\right) / \epsilon_{1}}$.

\subsection{Perpendicual polarization (TM)}

Now, we consider an incident monochromatic EM wave perpendicularly polarized incident on the elliptical anisotropic plasma layer given as

$$
E_{0 i}=E_{i} \hat{e}_{y} \exp \left[-j k_{i} z\right]
$$

The reflected and refracted wave vectors are obtained from the anisotropic plasma elliptical layer and the PEC elliptical reflector are given by as above

$$
p_{1}=-\hat{e}_{x} \sin 2 \alpha-\hat{e}_{z} \cos 2 \alpha
$$

$$
\begin{gathered}
q_{1}=-\hat{e}_{x} \sin \alpha\left(-\cos \alpha+\sqrt{n^{2}-\sin ^{2} \alpha}\right) \\
-\left(\cos \alpha\left(-\cos \alpha+\sqrt{n^{2}-\sin ^{2} \alpha}\right)+1\right) \hat{e}_{z} \\
p_{1}=-\hat{e}_{x} \sin 2 \alpha-\hat{e}_{z} \cos 2 \alpha \\
p_{1}=-\hat{e}_{x} \sin 2 \alpha-\hat{e}_{z} \cos 2 \alpha
\end{gathered}
$$

where $K_{4}=\sqrt{\left(2+n\left(-1+2(-1+n) n+n \cos \left(2 \alpha-2 \theta_{0}\right)\right) / 2\right.}$, $K_{3}=\sqrt{\left(-1+2 n^{2}+\cos 2 \alpha\right) / 2}$, and $n=\sqrt{\epsilon_{3}}$.

\section{GEOMETRICAL OPTICS FIELD}

The Hamilton's equations of the wave rays are given by

$$
\frac{d x}{d \tau}=p_{x}, \quad \frac{d z}{d \tau}=p_{z}, \quad \frac{d p_{x}}{d \tau}, \quad \text { and } \quad \frac{d p_{z}}{d \tau}=0 .
$$

The solutions of the Hamilton's equations can be written as

$$
\begin{gathered}
x=\xi_{0}+p_{1 x} \tau_{1}, \quad z=\zeta_{0}+p_{1 z} \tau_{1}, \\
p_{1 x}=p_{1 x}^{0}, \quad p_{1 z}=p_{1 z}^{0} \\
x=\xi_{0}+q_{1 x} \tau_{1}, \quad z=\zeta_{0}+q_{1 z} \tau_{1} \\
q_{1 x}=q_{1 x}^{0}, \quad q_{1 z}=q_{1 z}^{0},
\end{gathered}
$$

The Jacobian associated with waves reflected by the elliptical plasma layer is obtained as

$$
J_{1}\left(\tau_{1}\right)=\frac{D_{1}\left(\tau_{1}\right)}{D_{1}(0)}=1-\frac{2 a^{2} \tau_{1}}{R_{1} R_{2} \zeta_{0}}
$$

The Jacobian associated with waves refracted by an elliptical plasma layer with parallel polarization is obtained

$$
\begin{aligned}
& J_{2}\left(\tau_{2}\right)=\frac{D_{2}\left(\tau_{2}\right)}{D_{2}(0)} \\
& =1-\frac{a^{2} \tau_{2}}{K_{2}^{2} \sec \alpha R_{1} R_{2} \zeta_{0}}\left[2 K_{2}\left(2 K_{2}^{2}+n^{2}\right)+\right. \\
& \left.\quad n\left(4 K_{2}^{2}+n\right) \cos \alpha-n^{2}\left(2 K_{2} \cos 2 \alpha+\cos 3 \alpha\right)\right]
\end{aligned}
$$

Similarly, the Jacobian associated with waves refracted by the plasma layer with perpendicular polarization is obtained [29]-[33] as

$$
\begin{aligned}
& J_{2}\left(\tau_{2}\right)=\frac{D_{2}\left(\tau_{2}\right)}{D_{2}(0)} \\
& =1-\frac{a^{2} \tau_{2}}{K_{4}^{2} \sec \alpha R_{1} R_{2} \zeta_{0}}\left[2 K_{4}\left(2 K_{4}^{2}+n_{1}^{2}\right)+\right. \\
& \left.\quad n\left(4 K_{4}^{2}+n_{1}\right) \cos \alpha-n_{1}^{2}\left(2 K_{4} \cos 2 \alpha+\cos 3 \alpha\right)\right]
\end{aligned}
$$

The field expressions for the reflected and transmitted rays out of the anisotropic plasma layer in geometric optics are

$$
\begin{gathered}
E_{r}(x, z)=E_{i}\left[J_{1}\left(\tau_{1}\right)\right]^{-\frac{1}{2}} \exp \left[-j k\left(\Psi_{0}+\tau_{1}\right)\right] \\
E_{t}(x, z)=E_{i}\left[J_{2}\left(\tau_{2}\right)\right]^{-\frac{1}{2}} \exp \left[-j k\left(\Psi_{0}+\tau_{2}+t\right)\right]
\end{gathered}
$$

where $\Psi_{0}=\zeta_{0}, \tau_{1}$ and $\tau_{2}$ are parameters along the rays reflected and transmitted out of the elliptical anisotropic plasma layer to the focal point, respectively, and $t=\sqrt{\left(\xi_{0}-\xi\right)^{2}+\left(\zeta_{0}-\zeta\right)^{2}}$ is the distance between $P\left(\xi_{0}, \zeta_{0}\right)$ and $Q(\xi, \zeta)$. It is observed that the GO reflected and transmitted fields become infinity at the caustic or focal points as expected when $J(\tau)=0$. The expression that is valid at the focal point is derived using the Maslov's method. The exact location of caustic point is at $J(\tau)=0$.

\subsection{Field expressions in focal region for parallel (TM) polarization}

According to Maslov's method, the field expressions in the focal region can be written as

$$
\begin{aligned}
E^{r}(r)=\sqrt{\frac{k}{j 2 \pi}} \int_{-\infty}^{\infty} E_{t}(x, z)\left[\frac{D(\tau)}{D(0)} \frac{\partial\left(p_{z}\right)}{\partial(z)}\right]^{-\frac{1}{2}} . \\
\quad \exp \left\{-j k\left[S_{0}+\tau-z\left(p_{x}, z\right) p_{z}+p_{z} z\right]\right\} d p_{z}
\end{aligned}
$$

Integrands for the reflected and transmitted fields from the plasma layer are obtained, respectively, as

$$
\begin{gathered}
I_{1}=\frac{D\left(\tau_{1}\right)}{D(0)} \frac{\partial\left(p_{1 x}\right.}{\partial(x)}=-\frac{(a-d)^{2} \sin ^{2}(2 \alpha)}{R_{1} R_{2} \zeta_{0}} \\
I_{2}=\frac{D\left(\tau_{2}\right)}{D(0)} \frac{\partial\left(p_{2 x}\right.}{\partial(x)}=-\frac{(a-d)^{2} \cos \alpha \sin ^{2} \alpha}{K_{1}^{2} R_{1} R_{2} \zeta_{0}} . \\
\left(K_{2}+n \cos \alpha\right)\left(2 K^{2}+n+4 n K_{2} \cos \alpha+n \cos 2 \alpha\right)
\end{gathered}
$$

The phase functions of the integrals for the reflected and transmitted fields from the plasma layer are obtained, respectively, as

$$
\begin{aligned}
S_{1}=S_{0}+\tau_{1}-z\left(x, p_{z}\right) p_{z}+p_{x} x= \\
\\
\frac{(a-d)^{2} \cos \alpha\left(r \cos \theta+\cos 2 \alpha_{1}\right)}{\sqrt{c^{2} \sin ^{2} \alpha-(b-d)^{2}}} \\
-\frac{(b-d)^{2} \sin \alpha\left(r \sin \theta+\sin 2 \alpha_{1}\right)}{\sqrt{c^{2} \sin ^{2} \alpha-(b-d)^{2}}} \\
+r \sin \theta \cos (2 \alpha-\theta)
\end{aligned}
$$




$$
\begin{aligned}
S_{2} & =S_{0}+\tau_{2}+t-z\left(x, p_{z}\right) p_{z}+p_{x} x \\
& =\zeta_{0}-\xi_{0} q_{2 x}-\zeta_{0} q_{2 z}-x q_{2 x}+z q_{2 z}+\xi_{0} x+\zeta_{0} z
\end{aligned}
$$

where $x=r \sin \theta, \xi_{0}=-(b-d)^{2} \sin \alpha / \sqrt{c^{2} \sin ^{2} \alpha-(b-d)^{2}}$, $\zeta_{0}=-(a-d)^{2} \cos \alpha / \sqrt{c^{2} \sin ^{2} \alpha-(b-d)^{2}}, z=r \cos \theta$, $q_{2 x}=-\sin \alpha\left(K_{2}+n_{1} \cos \alpha\right)$ and $q_{2 z}=-K_{2} \cos \alpha+n_{1} \sin ^{2} \alpha$.

The initial value of the reflected field is related to the incident field by

$$
E_{r}(x, z)=-E_{i}-2\left(E_{i} \cdot N\right) N
$$

Using Eqs. (25), (27) and (30) into Eq. (24) and changing the variable $p_{1 z}$ to $\alpha$, given in Eq. (8), yields the final reflected field expression in components along the focal region

$$
\begin{aligned}
& \frac{E_{x}(x, z)}{E_{i}}= \\
& \quad \sqrt{\frac{k}{\pi}} \int_{-\frac{l}{2}}^{\frac{l}{2}} \frac{1-n}{1+n} \sqrt{\frac{R_{1} R_{2}\left((b-d)^{2}-\xi^{2}\right)}{\zeta_{0}(b-d)^{2}}} e^{-j k\left(S_{1}\right)} d \alpha
\end{aligned}
$$

The initial value of the transmitted field is related with the incident field by the relation

$$
E_{t}(x, z)=\tilde{T} \cdot E_{i}=\left[T_{\|} i_{\|}^{t} i_{\|}^{i}+T_{\perp} i_{\perp}^{t} i_{\perp}^{i}\right] \cdot E_{i}
$$

The unit vector corresponding to these components are obtained using the following relations:

$$
\begin{aligned}
& i_{\perp}^{i}=\frac{p^{i} \times n}{\left|p^{i} \times n\right|}=\hat{e}_{y}, i_{\|}^{i}=i_{\perp}^{i} \times p^{i}=\hat{e}_{x}, i_{\perp}^{t}=\frac{q^{i} \times n}{\left|q^{i} \times n\right|=\hat{e}_{y}}, \\
& i_{\|}^{t}=i_{\perp}^{i} \times q^{i}=\left(-K_{2} \cos \alpha+n_{1} \sin ^{2} \alpha\right) \hat{e}_{x} \\
& \quad-\left(K_{2} \sin \alpha+n_{1} \cos \alpha \sin \alpha\right) \hat{e}_{z}, \\
& T_{\|}=\frac{2 n_{1} \cos \alpha}{\cos \alpha+n_{1} \sqrt{1-n_{1}^{2} \sin ^{2} \alpha}}, T_{\perp}=\frac{2 n_{1} \cos \alpha}{n_{1} \cos \alpha+\sqrt{1-n_{1}^{2} \sin ^{2} \alpha}} .
\end{aligned}
$$

Again, using Eqs. (26), (29) and (33) into (24) and changing the variable $p_{2 z}$ to $\alpha$, given in Eq. (10), yields the final transmitted field expression in components form

$$
\begin{aligned}
& \frac{E_{x}(x, z)}{E_{i}}=\sqrt{\frac{2 k f}{\pi}} \int_{-l / 2}^{l / 2} E_{t x} \sqrt{\frac{K_{2} R_{2}}{\cos \alpha q_{2 x}} \frac{d \xi_{0}}{d \alpha} \frac{d q_{2 z}}{d \alpha}} e^{-j k S_{2}} d \alpha \\
& \frac{E_{z}(x, z)}{E_{i}}=\sqrt{\frac{2 k f}{\pi}} \int_{-l / 2}^{l / 2} E_{t z} \sqrt{\frac{K_{2} R_{2}}{\cos \alpha q_{2 x}} \frac{d \xi_{0}}{d \alpha} \frac{d q_{2 z}}{d \alpha}} e^{-j k S_{2}} d \alpha
\end{aligned}
$$

where

$$
\begin{aligned}
& E_{t x}=\frac{2 n_{1} \cos \alpha\left(-K_{2} \cos \alpha+n_{1} \sin 2 \alpha\right)}{n_{1} \cos \alpha+\sqrt{1-n_{1}^{2} \sin ^{2} \alpha}}, \\
& E_{t z}=\frac{n_{1} \sin 2 \alpha\left(-K_{2}+n_{1} \cos \alpha\right)}{n_{1} \cos \alpha+\sqrt{1-n_{1}^{2} \sin ^{2} \alpha}}
\end{aligned}
$$

where $l$ is the angle which the reflector subtends with the aperture.

\subsection{Field expressions in focal region for perpendicular (TM) polarization}

By using similar procedure, the final reflected field expression for the perpendicular polarization valid along the focal region is

$$
\begin{aligned}
& \frac{E_{y}^{r}(x, z)}{E_{i}} \\
& \quad=\sqrt{\frac{k}{\pi}} \int_{-l / 2}^{l / 2} \frac{1-n}{1+n} \sqrt{\frac{R_{1} F_{2}\left((b-d)^{2}-\xi^{2}\right)}{\zeta_{0}(b-d)^{2}}} e^{-j k\left(S_{1}\right)} d \alpha
\end{aligned}
$$

Again using Eqs. (19) and (21) into Eq. (24) and changing the variable $p_{2 z}$ to $\alpha$ in Eq. (10), yields the final finite transmitted field expression valid along the focal region as

$$
\begin{aligned}
& \frac{E_{y}^{t}(x, z)}{E_{i}}=\frac{1}{4 j \pi} . \\
& \quad \int_{-\frac{l}{2}}^{\frac{l}{2}} \frac{2 n \cos \alpha}{n \cos \alpha+\sqrt{1-n^{2} \sin ^{2} \alpha}} \sqrt{\frac{1}{q_{2 x}} \frac{d q_{2 z}}{d \alpha} \frac{d \xi_{0}}{d \alpha} \frac{K_{4}}{\cos \alpha} .} \\
& e^{-j k\left(\zeta_{0}-\xi_{0} q_{2 x}-\zeta_{0} q_{2 x}-x q_{2 x}+z q_{2 z}+\xi_{0} x+\zeta_{0} z\right)} d \alpha
\end{aligned}
$$

where $\xi_{0}, \zeta_{0}, x$ and $z$, are defined in Eq. (28) and $q_{2 x}$ and $q_{2 z}$ are given in Eq. (16)

\section{HUYGENS-KIRCHHOFF'S SOLUTION}

To validate the formulation derived in the preceding section, the fields are obtained using the following formula implied by Huygens-Kirchhoff's principle [27, 28]

$$
E(x, z)=\sqrt{\frac{k}{j 2 \pi}} \int_{-\infty}^{\infty} \frac{e^{-j R}}{R} E_{0}\left(\xi_{0}, \zeta_{0}\right) \exp \left(-j k S_{0}\right) d \xi_{0}
$$

where $R=q_{1 x}\left(x-\xi_{0}\right)+q_{1 z}\left(z-\zeta_{0}\right)=\sqrt{\left(x-\xi_{0}\right)^{2}+c_{2}^{2}}$ and $E_{0}\left(\xi_{0}, \zeta_{0}\right)=J^{-\frac{1}{2}}$. Therefore

$$
E(x, z)=\sqrt{k j 2 \pi} \int_{-l / 2}^{l / 2} \frac{J^{-\frac{1}{2}}}{R} \exp \left[-j k\left(S_{0}+R\right] d \xi_{0}\right.
$$

\section{RESULTS AND DISCUSSIONS}

The reflected and transmitted EM waves due to a normally incident plane wave on an elliptical metal reflector with anisotropic plasma layer on its surface are depicted in this section. The frequency of the incident EM wave is taken in the microwave region. First, the results are compared with the Huygens-Kirchhoff approximation to check the correctness of the developed analytical formulations. Figure 2 shows the comparison of the numerical results using Maslov's method (solid line) and using Kirchhoff's approximation (dashed line) which confirms that the agreement is good.

Figure 3(a)-(b) illustrate the refelcted field intensity distribution from the elliptical reflector in the presence of the anisotropic plasma layer along the focal points along $k z$ for parallel polarization and perpendicular polarization respectively, at $k a=60, k b=6, n_{e}=6 \cdot 10^{12} \mathrm{~m}^{-3}, \omega=3.14 \cdot 10^{8} \mathrm{~Hz}$, $\omega_{c}=0.3 \cdot 10^{8} \mathrm{~Hz}$, and $v=1.5 \cdot 10^{5} \mathrm{~Hz}$, for different values 


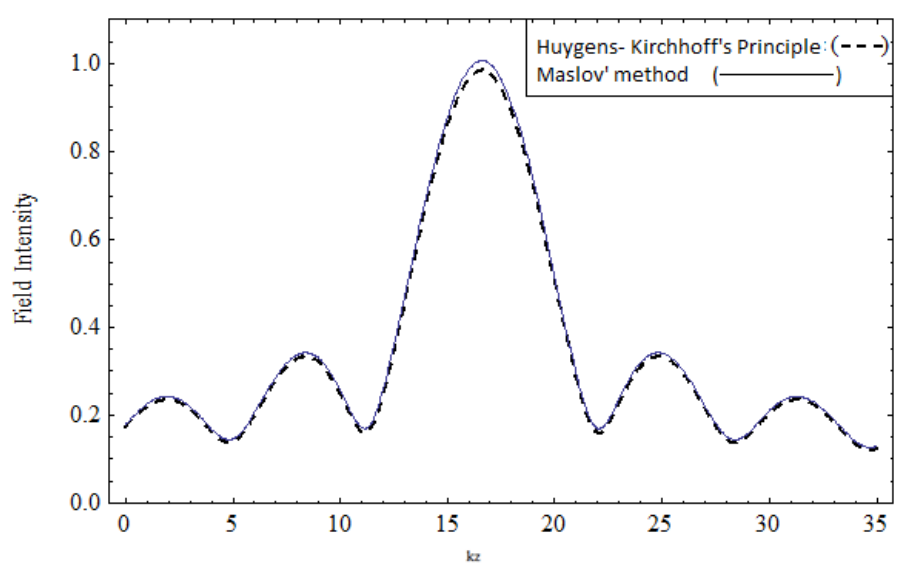

FIG. 2 The normalized field intensity by Maslov's method (solid line) and by Kirchhoff's integral (dashed line).
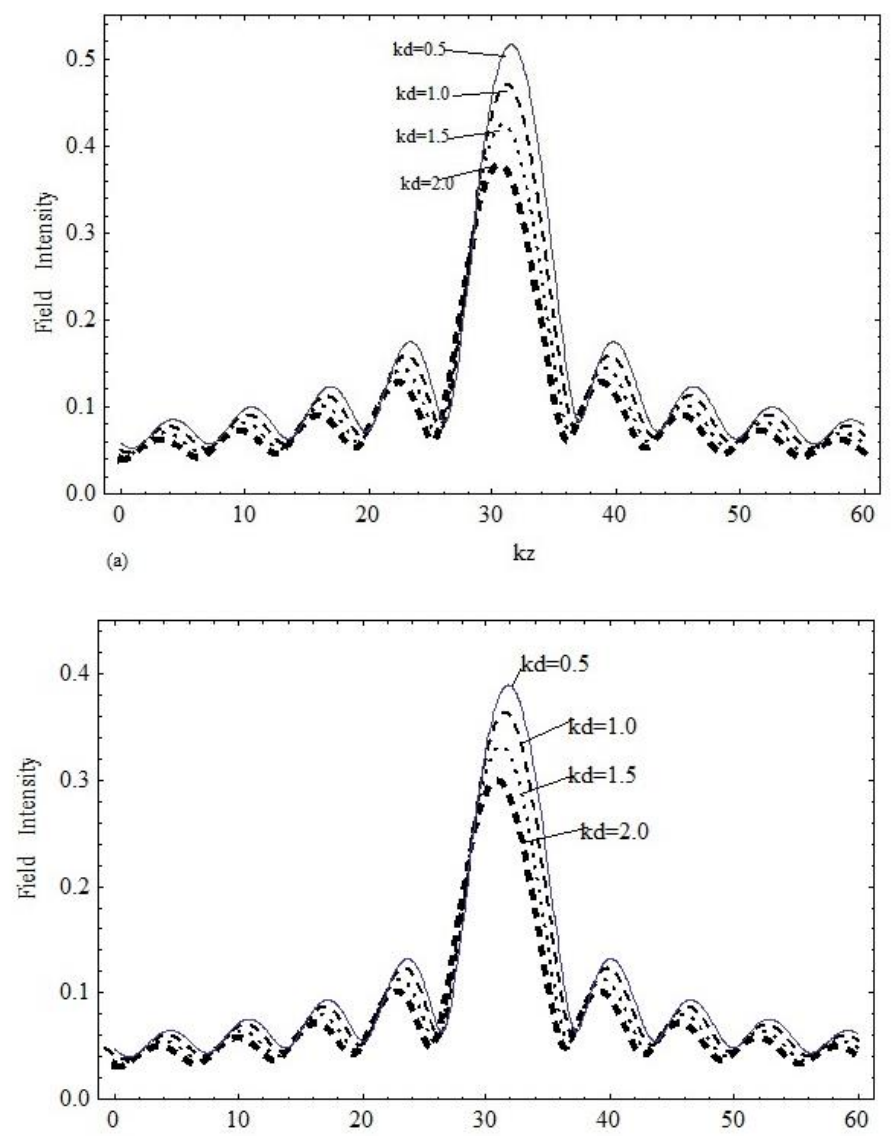

(b)

$\mathrm{kz}$

FIG. 3 The reflected field intensity distribution with the thickness of the anisotropic plasma layer using Maslov's method along the z-axis.

of plasma layer thickness. The value of the reflected field intensity decreases and the location of the maximum reflected field intensity are displaced along the $z$-axis away from the reflector curved-surface at higher values of the plasma layer thickness for both parallel and perpendicular polarizations.

Figure 4(a)-(b) shows the comparison of the transmitted field intensity distribution from the elliptical reflector in the presence of the anisotropic plasma layer along the focal points along $k z$ for parallel polarization and perpendicular polarization respectively, at $k a=60, k b=6, n_{e}=6 \cdot 10^{12} \mathrm{~m}^{-3}$, $\omega_{c}=0.3 \cdot 10^{8} \mathrm{~Hz}, v=1.5 \cdot 10^{5} \mathrm{~Hz}$, and $\omega=3.14 \cdot 10^{8} \mathrm{~Hz}$ for different plasma layer thickness values. The transmitted field intensity decreases in magnitude but with some displace-
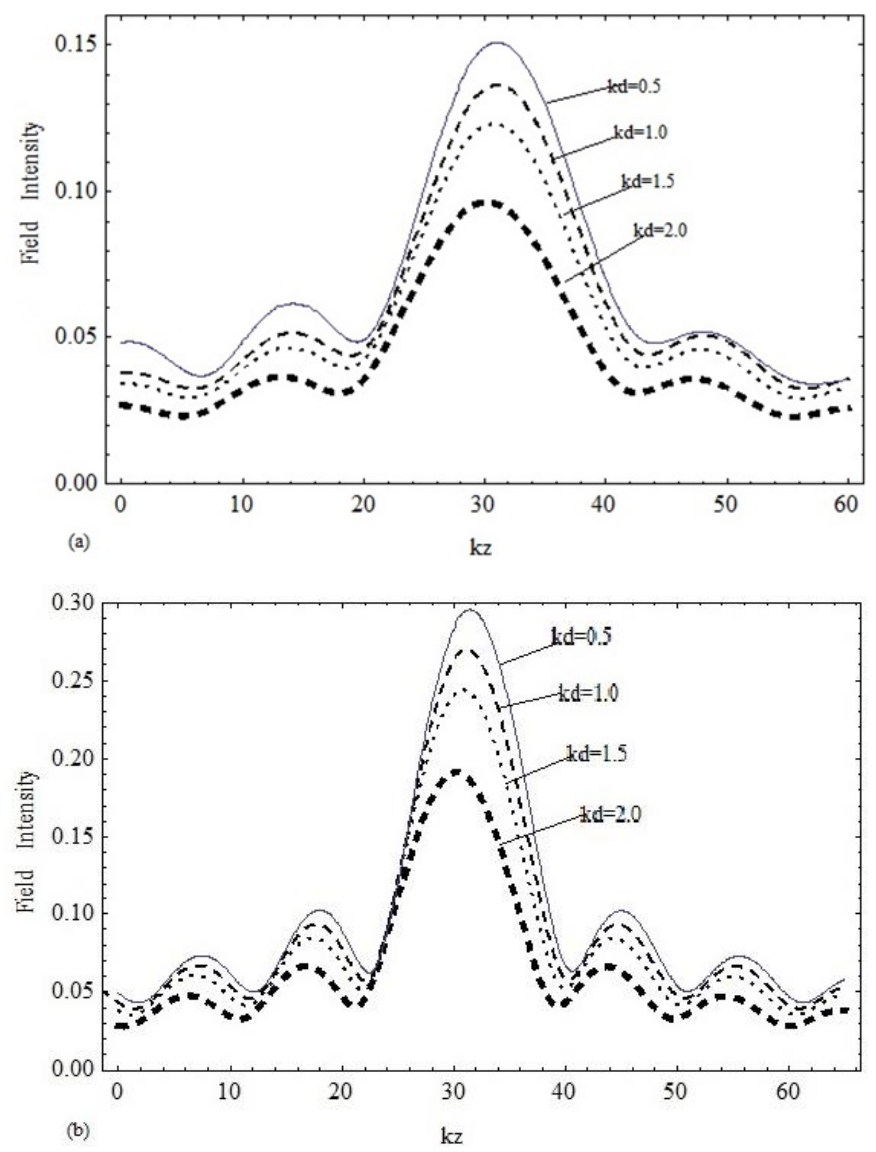

FIG. 4 The transmitted field intensity distribution for different values of the plasma layer thickness with the z-axis (a) parallel polarization (b) perpendicular polarization.

ment away from the reflector curved-surface along the $z$-axis at higher values of the anisotropic plasma layer thickness. The number of side lobes and the focus of the main lobe of the transmitted field are more than that of the reflected field intensity.

Figures 5 to 6 demonstrate, respectively, the effects of the plasma electron density, and the cyclotron frequency on the transmitted field distribution from the anisotropic plasma layer. Figures 5(a)-(b) shows the transmitted field intensity versus $k z$ for different values of the anisotropic plasma electron density with parallel polarization and perpendicular polarization incident waves. These figures have been plotted for a constant foci radius of the metallic elliptical reflector $k a=60, k b=6$, an incident-wave frequency $\omega=3.14 \cdot 10^{8} \mathrm{~Hz}, v=1.5 \cdot 10^{5} \mathrm{~Hz}$, and $k d=0.1$. It is clearly observed that if the electron density of the plasma layer decreases the value of the transmitted field intensity increases for both cases and the transmitted field intensity of the parallel polarization is sharp as compared to that of the perpendicular polarization. The case of electron plasma density $n_{e}=0$ corresponds to a bare metallic reflector. The level of the side lobes of the plasma coated reflector is higher than that of the metallic reflector whereas their main lobes are similar. By varying the plasma density in the plasma layer, the phase of the reflected signal can be altered. The steering and focusing of the reflector may occur as desired. The plasma-coated reflector can be more stealth at high frequencies.

Figure 6(a)-(b) represents the changes of the transmitted field 

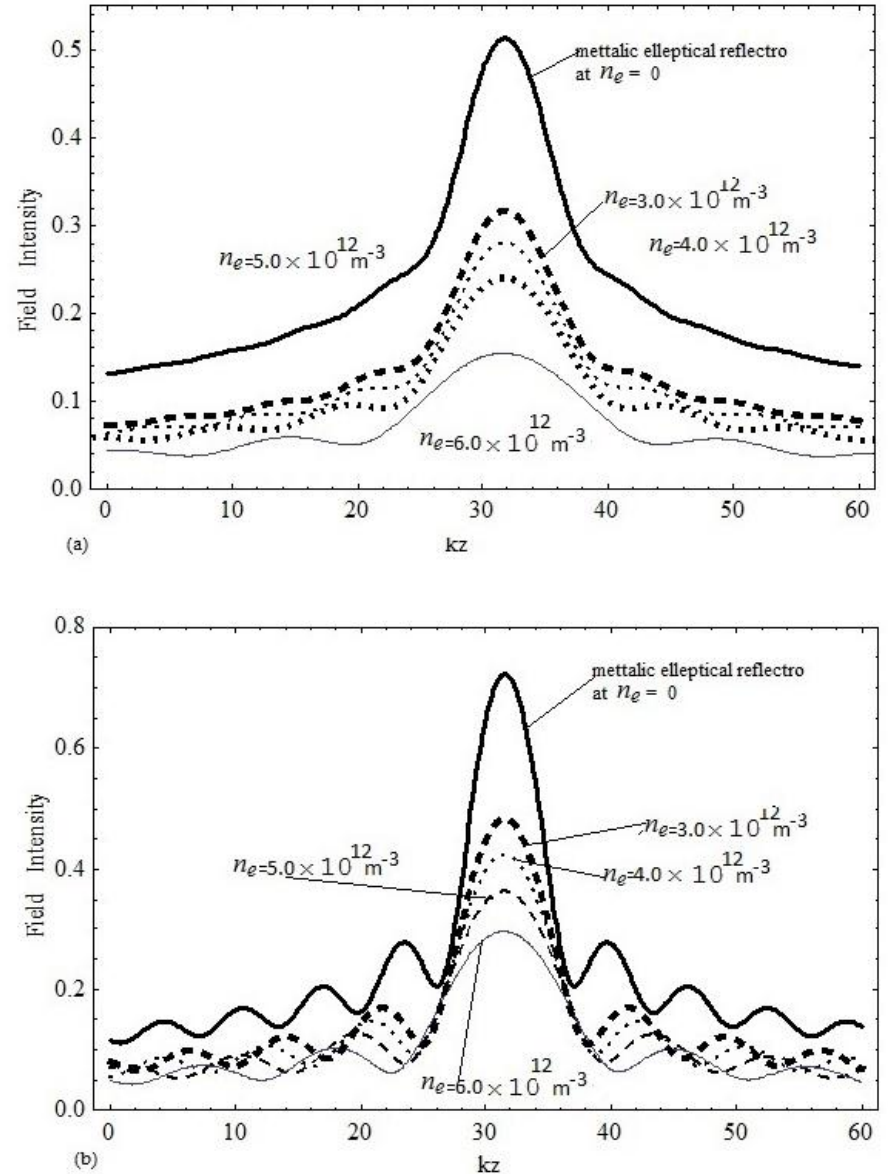

FIG. 5 The transmitted field intensity distribution for different values of the plasma electron density with the z-axis (a) parallel polarization (b) perpendicular polarization.

intensity versus $k x$ and $k z$ for parallel polarization incident waves, respectively, at different values of the cyclotron frequency $\omega_{c}$ by considering a constant value of foci radius of the metallic elliptical reflector $k a=60, k b=6$, plasma layer thickness $k d=0.1, v=1 \cdot 10^{5} \mathrm{~Hz}$, and plasma electron density $n_{e}=6 \cdot 10^{1} 2 \mathrm{~m}^{-3}$. In this demonstration, if the cyclotron frequency $\omega_{c}$ decreases, the value of the transmitted field intensity increases along both axes.

Figure $7(a)-(b)$ represents the changes of the transmitted field intensity versus $k z$ for parallel polarization and perpendicular polarization incident waves, respectively, at different values of the effective collisional frequency $v$ by considering a constant value of foci radius of the metallic elliptical reflector $k a=60, k b=6$, plasma layer thickness $k d=0.1, \quad \omega_{c}=0.3 \cdot 10^{8} \mathrm{~Hz}$, and plasma electron density $n_{e}=6 \cdot 10^{12} \mathrm{~m}^{-3}$. In this demonstration, if the effective collisional frequency $v$ increases, the value of the transmitted field intensity increases slightly and a non-uniformity of the field distribution along focal point is observed. The effects of the collisional frequency on field distribution for perpendicular polarization case are minima similar to results reported in [11].

\section{CONCLUSIONS}

This paper presents theoretical analyses of the EM fields in the focal region of a metal elliptical reflector covered with an anisotropic plasma layer under both parallel and perpen-

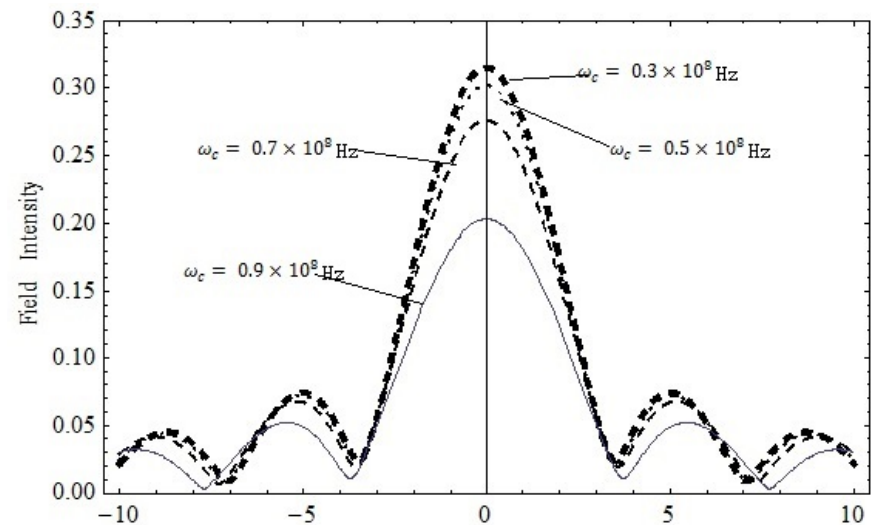

(a)

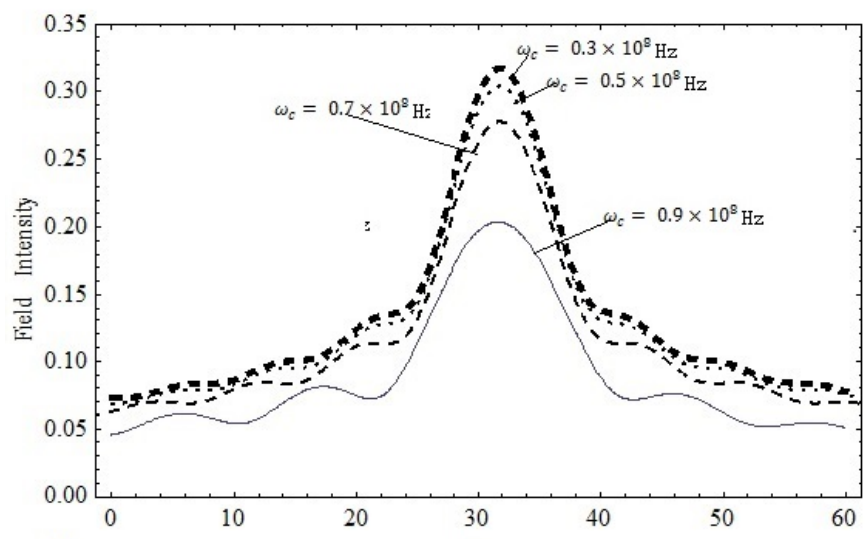

(b)

FIG. 6 The transmitted field intensity distribution for different values of the cyclotron frequency with the $x$-axis for parallel polarization (a) $x$-axis (b) $z$-axis.

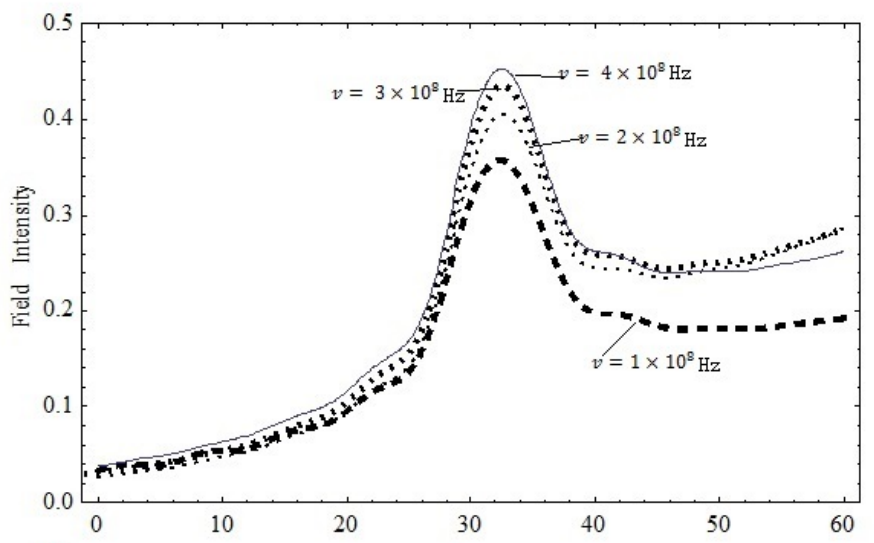

(a)

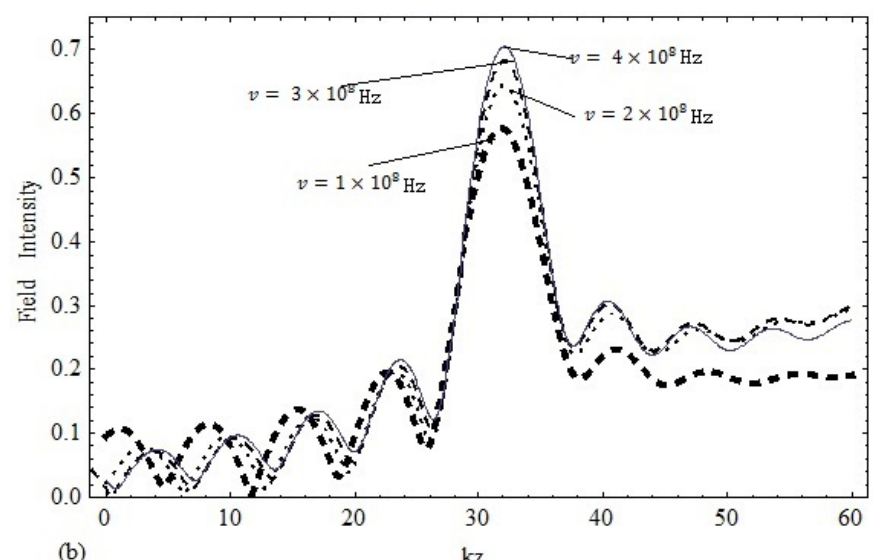

FIC. 7 The transmitted field intensity distribution for different values of the collisional frequency with the z-axis (a) parallel polarization (b) perpendicular polarization. 
dicular polarized-wave incidence. Accurate analytical expressions for the field intensity distributions along the focal line were derived using Maslov's method, which is an efficient procedure based on the asymptotic ray theory (ART) and the Fourier transform method. The reflected and the transmitted fields out of the anisotropic plasma layer were found to be in a good agreement to those obtained using Kirchhoff's approximation. The effects of the anisotropic plasma layer thickness, the plasma frequency; the cyclotron frequency, and the effective collisional frequency on the transmitted and the reflected energy distribution were examined. The focal point is slightly displaced away from the reflector surface along the $z$ axis at higher values of the plasma layer thickness. The plasma electron density and the cyclotron frequency have opposite influence on the value-levels of the transmitted field intensities in the focal region of the elliptical reflector as illustrated in Figure 5 and 6. A uniform field intensity distribution is more manifested at lower values of the effective collisional frequency. For the magnetized plasma case, the effect of the collisional frequency variations on the field uniformity is much higher than its effect in the un-magnetized plasma case.

\section{ACKNOWLEDGEMENTS}

The authors would like to extend their sincere appreciation to the Deanship of Scientific Research (DSR) at King Saud University for its funding of this research through the Research Group Project no RG-1436-001.

\section{References}

[1] D. R. Nicholson, Introduction to plasma theory (Cambridge University Press, Cambridge, 1983).

[2] V. Smilyanskii, "Propagation of an electromagnetic wave across a magnetic field in a parabolic plasma layer," J. Appl. Mech. Tech. Phy. 12, 366-371, 1971.

[3] A. Chaffar, M. Yaqoob, M. A. Alkanhal, M. Sharif, and Q. Naqvi, "Electromagnetic scattering from anisotropic plasma-coated perfect electromagnetic conductor cylinders," AEU-Int. J. Electron. C. 68, 767-772 (2014).

[4] A. Chaffar, and M. A. Alkanhal, "Fields in the focal region of an elliptical reflector coated with an unmagnetized plasma layer," Wave. Random Complex 25, 405-415 (2015).

[5] M. Laroussi, and J. Reece Roth, "Numerical calculation of the reflection, absorption, and transmission of microwaves by a nonuniform plasma slab," IEEE T. Plasma Sci. 21, 366-372 (1993).

[6] I. Alexeff, T. Anderson, S. Parameswaran, E. Michael, J. Dhanraj, and M. Thiyagarajan, "Advances in plasma antenna design," Proceedings to IEEE International Conference on Plasma Science, 350-350 (2005).

[7] G. Kuz'min, I. Minaev, K. Rukhadze, V. Tarakanov, and 0 . Tikhonevich, "Reflector plasma array antennas," J. Commun. Technol. El. 57, 536-542 (2012).

[8] H. Ling, S.-W. Lee, P. Lam, and W. Rusch, "Focal shifts in parabolic reflectors," IEEE T. Antenn. Propag. 33, 744-748 (1985).

[9] H. Minnett, and B. Thomas, "Fields in the image space of symmetrical focusing reflectors," P. I. Electr. Eng. 115, 1419-1430 (1968).
[10] H. Ling, and S.-W. Lee, "Focusing of electromagnetic waves through a dielectric interface," JOSA A 1, 965-973 (1984).

[11] W. B. Dou, Z. L. Sun, and X. Q. Tan, "Fields in the focal space of symmetrical hyperbolic focusing lens," Prog. Electromagn. Res. 20, 213-226 (1998).

[12] B. Jazi, B. Davoudi-Rahaghi, M. R. Khajehmirzaei, and A. R. Niknam, "Energy distribution along the focal axis of a metallic cylindrical parabolic reflector covered with a plasma layer," IEEE T. Plasma Sci. 42, 286-292 (2014).

[13] A. Chaffar, M. Yaqoob, M. A. Alkanhal, S. Ahmed, Q. Naqvi, and M. Kalyar, "Scattering of electromagnetic wave from perfect electromagnetic conductor cylinders placed in un-magnetized isotropic plasma medium," Optik 125, 4779-4783 (2014).

[14] Z.-C. Yuan, and J.-M. Shi, "Electromagnetic reflection of conductive plane covered with double inhomogeneous plasma slabs," Int. J. Appl. Electrom. 42, 623-628 (2013).

[15] A. Chayekhloo, and A. Abdolali, "Use of collisional plasma as an optimum lossy dielectric for wave absorption in planar layers, analysis, and application," IEEE T. Plasma Sci. 42, 1999-2006 (2014).

[16] A. Ghaffar, and M. Alkanhal, "Electromagnetic field intensity distribution along focal region of a metallic circular reflector covered with a plasma layer," J. Eur. Opt. Soc.-Rapid 10, 15014 (2015).

[17] A. Niknam, M. Khajehmirzaei, B. Davoudi-Rahaghi, Z. Rahmani, B. Jazi, and A. Abdoli-Arani, "Electromagnetic modeling of the energy distribution of a metallic cylindrical parabolic reflector covered with a magnetized plasma layer," Phys. Plasmas 21, 073107 (2014).

[18] D. Tang, A. Sun, X. Qiu, and P. K. Chu, "Interaction of electromagnetic waves with a magnetized nonuniform plasma slab," IEEE T. Plasma Sci. 31, 405-410 (2003).

[19] F. Jin, H. Tong, Z. Shi, D. Tang, and P. K. Chu, "Effects of external magnetic field on propagation of electromagnetic wave in uniform magnetized plasma slabs," Comput. Phys. Commun. 175, 545-552 (2006).

[20] A. Ghaffar, and M. A. Alkanhal, "Electromagnetic waves in uniaxial anisotropic chiral waveguides in magnetized plasma," Wave. Random Complex 25, 323-333 (2015).

[21] D.-W. Duan, and Y. Rahmat-Samii, "Diffraction shaping of reflector antennas with elliptical apertures and circular feed," in Proceedings to Antennas and Propagation Society International Symposium, 46-49 (IEEE, Seattle, 1994).

[22] S. Skyttemyr, "An elliptical aperture dual shaped reflector antenna for SNG applications," in Proceedings to Antennas and Propagation Society International Symposium, 558-561 (IEEE, Salt Lake City, UT, USA, 2000).

[23] A. Densmore, and Y. Rahmat-Samii, "Three-parameter elliptical aperture distributions for difference patterns by particle swarm optimization," in Proceedings to Antennas and Propagation Society International Symposium (APSURSI), 47-48 (IEEE, Memphis, 2014).

[24] Y. Rahmat-Samii, "Jacobi-Bessel analysis of reflector antennas with elliptical apertures," IEEE T. Antenn. Propag. 35, 1070-1074 (1987).

[25] C. Thomson, and C. Chapman, "An introduction to Maslov's asymptotic method," Geophys. J. Int. 83, 143-168 (1985).

[26] R. W. Ziolkowski, and G. A. Deschamps, "Asymptotic evaluation of high-frequency fields near a caustic: an introduction to Maslov's method," Radio Sci. 19, 1001-1025 (1984). 
[27] K. Hongo, Y. Ji, and E. Nakajima, "High-frequency expression for the field in the caustic region of a reflector using Maslov's method," Radio Sci. 21, 911-919 (1986).

[28] K. Hongo, and Y. Ji, "High-frequency expression for the field in the caustic region of a cylindrical reflector using Maslov's method," Radio Sci. 22, 357-366 (1987).

[29] A. Chaffar, S. Ahmed, and Q. Naqvi, "Electromagnetic field in the caustic region of a spherical reflector under oblique incidence using Maslov's method," Int. J. Appl. Electrom. 40, 1-17 (2012).

[30] A. Chaffar, M. Shoaib, N. Mehmood, M. Naz, M. Azam, and Q. Naqvi, "Electromagnetic field diffraction of a paraboloidal mirror under oblique incidence," Int. J. Appl. Electrom. 41, 479-494 (2013).
[31] A. Chaffar, M. Arif, Q. A. Naqvi, and M. A. Alkanhal, "Radiation properties of a uniaxial chiral quadratic inhomogeneous slab under oblique incidence," Optik 125, 1589-1597 (2014).

[32] A. Chaffar, M. Sharif, Q. Naqvi, M. Alkanhal, F. Khalid, and S. Shukurullah, "Focusing of electromagnetic wave from quartic inhomogeneous chiro-slab," Appl. Comput. Electrom. 29, 478-485 (2014).

[33] A. Chaffar, H. Shahbaz Ahmad, and Q. Naqvi, "Analysis of electromagnetic field transmitted by a quadratic inhomogeneous slab composed of chiral medium," Int. J. Appl. Electrom. 42, 613-622 (2013). 